\title{
Intrahepatic cholangiocarcinoma in an obese patient qualified for laparoscopic bariatric surgery - a case study
}

\author{
Maciej Walędziak ${ }^{1}$, Anna Różańska-Walędziak ${ }^{2}$, Piotr K. Kowalewski ${ }^{1}$, Krzysztof Paśnik $^{1}$ \\ ${ }^{1}$ Department of General, Oncological, Metabolic and Thoracic Surgery, Military Institute of Medicine, Warsaw, Poland \\ ${ }^{2} 2^{\text {nd }}$ Department of Obstetrics and Gynecology, Medical University of Warsaw, Warsaw, Poland
}

Videosurgery Miniinv 2018; 13 (2): 257-259

DOI: https://doi.org/10.5114/wiitm.2018.72751

\begin{abstract}
Intrahepatic cholangiocarcinoma is a rare type of biliary tract malignancy, seldom found resectable at diagnosis, the 5-year survival rate depending on the possibility of complete surgical excision. In most cases it is only accidentally found in the early stage. The report presents a case of a 58-year-old obese male patient qualified for laparoscopic Roux-en-Y gastric bypass. During general inspection of the abdominal cavity, a tumor of $10 \mathrm{~mm}$ in diameter was found in the second hepatic segment and resected. Final pathology results showed low-grade intrahepatic cholangiocarcinoma without vascular invasion, with positive margins of incision (R1), classified as stage I. The abdominal computed tomography scan showed no evidence of metastatic disease. Two months later the patient underwent a laparoscopic partial hepatectomy. Pathological investigation showed focal bile duct hamartoma with no signs of malignancy. No adjuvant therapy was administered and no recurrence has been found to date.
\end{abstract}

Key words: obesity, bariatric surgery, cholangiocarcinoma.

\section{Introduction}

Obesity has become an important health problem with the number of patients increasing worldwide. More than two thirds of the American population have a body mass index (BMI) $\geq 25 \mathrm{~kg} / \mathrm{m}^{2}$ [1]. Overweight and obesity are associated with higher risk of all-cause mortality, [2] and multiple comorbidities including type 2 diabetes and cardiovascular diseases. It amplifies oncological risks in breast, lung, pancreatic, liver, colorectal, kidney, prostate, ovarian, endometrial and esophageal cancer [3]. In 2014 over 1500 bariatric procedures were performed in Poland [4].

Excess body weight also increases the risk of cholangiocellular carcinoma (CCA) [5], one of the most malignant cancers, with the lowest rate of 1-year survival. Cholangiocellular carcinoma is classified as intrahepatic, perihilar or extrahepatic, based on its location. Histologically, 90\% of CCAs are adenocarcinomas, while the average age of presentation is 50 years. There are several risk factors for CCA such as primary sclerosing cholangitis (PSC), chronic intraductal gallstones, liver-fluke infestation, choledochal (bile duct) cysts, Caroli disease (intrahepatic biliary cysts), bile duct adenoma, biliary papillomatosis, inflammatory bowel disease (ulcerative colitis), and chronic typhoid carriage. Chronic viral hepatitis B or $C$, diabetes mellitus, fatty liver disease, alcohol overuse, smoking and polymorphisms of genes may also be potential risk factors for CCA [6]. Patients with early onset of CCA present no typical symptoms [7]. Surgical treatment is the only potentially curative therapeutic option for intrahepatic cholangiocellular carcinoma (CCAs) [7]. 


\section{Case report}

A 58-year-old male patient with obesity was admitted for bariatric surgery. His BMI was $43.87 \mathrm{~kg} /$ $\mathrm{m}^{2}$, weight $138 \mathrm{~kg}$ and height $178 \mathrm{~cm}$. His highest measured BMI was $51.13 \mathrm{~kg} / \mathrm{m}^{2}$. He lost over $20 \mathrm{~kg}$ with diet and exercise during 6 months prior to the operation. The patient was a heavy smoker (more than 20 cigarettes per day for the previous 10 years) and suffered from hypertension, dyslipidemia, type 2 diabetes mellitus (T2DM), severe obstructive sleep apnea (OSA) and atrial fibrillation (AF). The patient disclaimed potential symptoms of CCA such as abdominal pain, night sweats, emesis, malaise, loss of appetite or pruritus. The patient had no history of abdominal surgery. There were no abnormalities found in pre-operative upper gastrointestinal tract endoscopy. Ultrasonography (USG) of the abdominal cavity did not show liver abnormalities or lesions. Routine laboratory testing (complete blood count, serum chemistry panel and liver function tests) did not reveal any abnormalities. Neither the antigen of the hepatitis B virus (HBV) nor hepatitis C virus antibodies (anti-HCV) were found.

Based on BMI, alimentary habits and co-morbidities, the patient was qualified for laparoscopic Rouxen- $Y$ gastric bypass (LRYGB). Once the abdomen was insufflated, the procedure started with an inspection of the abdominal cavity. A tumor of $10 \mathrm{~mm}$ in diameter was found in the second hepatic segment (according to Couinaud's classification). The tumor was resected and an intraoperative pathological analysis was performed. The result was non-conclusive and because of the suspected malignant character of the lesion, the operation was terminated without performing the bariatric procedure. The operation time was $50 \mathrm{~min}$, there were no postoperative complications, and the patient was discharged on the next day. Final pathology results showed low-grade intrahepatic cholangiocarcinoma without vascular invasion with positive margins of incision (R1). Additional immunohistochemical examinations presented the characteristics of tumor: CK7 +, CK19 +, CK20 -, hepatocytes -, p53 +, PAS -. The tumor was classified as stage I according to the American Joint Committee on Cancer and International Union Against Cancer staging system (AJCC/UICC). The patient was referred to an abdominal computed tomography (CT) scan, which showed no evidence of metastatic disease. The case was analyzed by a consulting oncological group and the patient was qualified for a laparoscopic partial hepatectomy, performed 2 months later by the same bariatric team, who included an oncological surgeon. The operation length was $105 \mathrm{~min}$, there were no postoperative complications, and on the second day after the operation the patient was discharged. Pathological investigation showed focal bile duct hamartoma with no signs of malignancy. No adjuvant therapy was administered. To date, 5 months after the original surgery, the patient continues to be in remission.

\section{Discussion}

Cholangiocellular carcinoma is the most common biliary tract malignancy [7]. Numerous studies have proven the association between obesity and intrahepatic CCA, which represents only $6-8 \%$ of all CCAs $[5,8]$. About $4 \%$ of benign incidental findings are discovered during bariatric surgery [9]. However, there were no reports about incidental findings of ICC during bariatric surgery before. Chang et al. [10] reported an association between diabetes and ICCA. According to Khan et al. [11], patients with suspected CCA should have combined magnetic resonance imaging (MRI) and magnetic resonance cholangiopancreatography (MRCP) or contrast-enhanced high resolution $\mathrm{CT}$. Ultrasonography offers specificity of $90 \%$, but sensitivity of only $50 \%$, and small tumors may be missed. Although surgical treatment may have curative potential, fewer than $1 / 3$ of ICCS are possible to resect at diagnosis [11]. In the present case, the resected tumor was classified as T1NOMO according to the TNM classification (tumor, nodes, metastasis) and stage I of the $7^{\text {th }}$ edition of the AJCC/UICC staging system [12]. In 2014, the Liver Cancer Study Group of Japan (LCSGJ) proposed a new staging system for mass-forming ICCS, providing better stratification of survival in patients who had undergone curative resection of ICCS [13]. The study showed 3- and 5-year survival rates of patients with tumors less than $2 \mathrm{~cm}$ in diameter, with no node involvement and no metastases, of $100 \%$ and $92,3 \%$ respectively. According to the CCA review presented by Ghouri et al., the 5 -year survival rate is $23-42 \%$ after $\mathrm{RO}$ resection versus $0 \%$ after $\mathrm{R}+$ resection [7]. For patients with non-resectable cancers, chemotherapy, local ablative therapies or even orthotropic liver transplantation can be considered. 


\section{Conclusions}

As obesity is associated with higher risk for cancerogenesis, every bariatric surgery procedure should start with meticulous peritoneal cavity investigation. Each tumor incidentally found should be resected with surgical margins to provide potential curative surgical treatment in case of malignancy. Each suspected specimen should undergo pathological examination.

\section{Conflict of interest}

The authors declare no conflict of interest.

\section{References}

1. National Center for Health Statistics. Health, United States, 2015: With Special Feature on Racial and Ethnic Disparities. Hyattsville, MD: National Center for Health Statistics, 2016.

2. Berrington de Gonzalez A, Hartge P, Cerhan JR, et al. Body-mass index and mortality among 1.46 million white adults. N Engl J Med 2010; 363: 2211-9. Erratum in: N EngI J Med 2011; 365: 869.

3. Guh DP, Zhang W, Bansback N, et al. The incidence of co-morbidities related to obesity and overweight: a systematic review and meta-analysis. BMC Public Health 2009; 9: 88.

4. Janik MR, Stanowski E, Paśnik K. Present status of bariatric surgery in Poland. Videosurgery Miniinv 2016; 11: 22-5.

5. Li JS, Han TJ, Jing N, et al. Obesity and the risk of cholangiocarcinoma: a meta-analysis. Tumor Biol 2014; 35: 6831-8.

6. Plentz RR, Malek NP. Clinical presentation, risk factors and staging systems of cholangiocarcinoma. Best Pract Res Clin Gastroenterol 2015; 29: 245-52.

7. Ghouri YA, Mian I, Blechacz B. Cancer review: cholangiocarcinoma. J Carcinog 2015; 14: 1.

8. Palmer WC, Patel T. Are common factors involved in the pathogenesis of primary liver cancers? A meta-analysis of risk factors for intrahepatic cholangiocarcinoma. J Hepatol 2012; 57: 69-76.

9. Walędziak M, Różańska-Walędziak A, Kowalewski PK, Janik MR, Paśnik K. Histopathological examination of tissue resected during bariatric procedures - to be done or not to be done? Videosurgery Miniinv 2017; 12: 135-9.

10. Chang JS, Tsai CR, Chen LT. Medical risk factors associated with cholangiocarcinoma in Taiwan: a population-based case-control study. PLoS One 2013; 8: e69981

11. Khan SA, Davidson BR, Goldin RD, et al. Guidelines for the diagnosis and treatment of cholangiocarcinoma: an update. Gut 2012; 61: 1657-69.

12. Edge S, Byrd DR, Compton CC, et al. AJCC Cancer Staging Manual ( $7^{\text {th }}$ ed). New York, Springer, 2010.

13. Uenishi T, Ariizumi S, Aoki T, et al. Proposal of a new staging system for mass-forming intrahepatic cholangiocarcinoma: a multicenter analysis by the Study Group for Hepatic Surgery of the Japanese Society of Hepato-Biliary-Pancreatic Surgery. J Hepatobiliary Pancreat Sci 2014; 21: 499-508.

Received: 8.05.2017, accepted: 23.09.2017. 